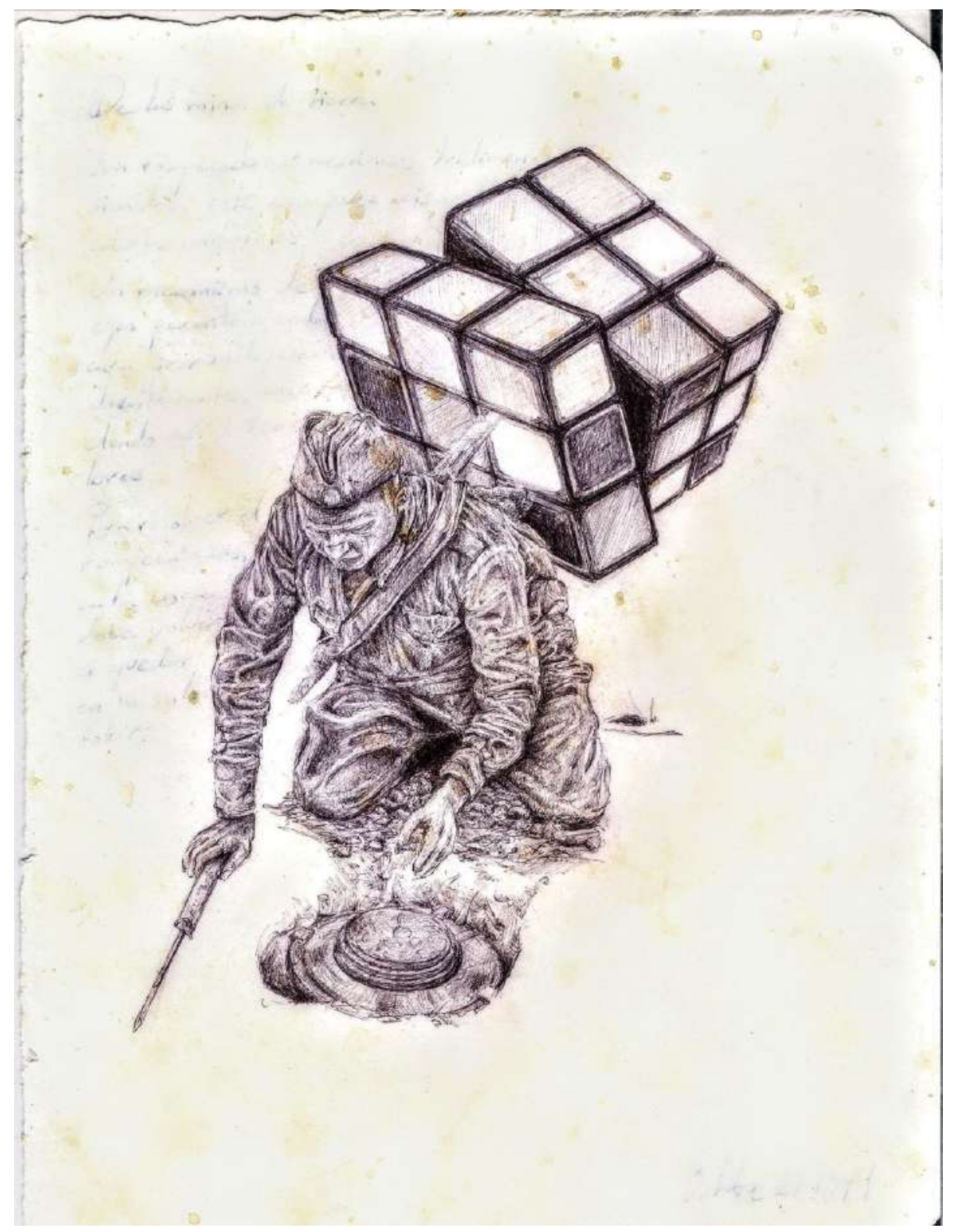

Sebastián Zea Quintero

Sin título

De la serie Del juego y el hombre Lápiz sobre papel

2017

Medellín 


\title{
La formalización minera en el Bajo Cauca antioqueño a través de las áreas de reserva especial. Una aproximación al diseño institucional desde el marco de análisis y desarrollo institucional ${ }^{*}$
}

\author{
Mario Alfonso Lopera González (Colombia)**
}

\section{Resumen}

El aumento en los precios del oro al despuntar el siglo xxı ha conducido a un incremento en la explotación aurífera; sin embargo, la mayor parte de la minería en el territorio nacional no cuenta con los mínimos legales. A la informalidad en la extracción aurífera se suman problemas como la sobreexplotación de los recursos, las externalidades ambientales, el financiamiento de grupos armados organizados al margen de la ley, entre otros. Así las cosas, las políticas públicas están Ilamadas a propender por mecanismos adecuados para la formalización minera. El artículo da cuenta de una implementación integral del marco de análisis y desarrollo institucional (ADI) - bloques universales de interacción, sintaxis Adico, tipología genérica de reglas - indagando por la viabilidad, desde la perspectiva de su diseño institucional, del mecanismo de las áreas de reserva especial (ARE) en el contexto del Bajo Cauca antioqueño. El artículo muestra la utilidad del marco ADI para el diagnóstico y diseño de políticas públicas sensibles al contexto de implementación, y evidencia las dificultades del mecanismo ARE en la subregión.

\section{Palabras clave}

Minería; Conflictos por Recursos Minerales; Formalización Minera; Análisis y Desarrollo Institucional; Sintaxis Adico; Colombia.

\footnotetext{
* Este artículo es producto del proceso de investigación de la maestría en Políticas Públicas, Facultad de Ciencias Económicas, Universidad de Antioquia, y apoyada con recursos del Centro de Investigaciones y Consultoría (CIC), 2017-II.

** Politólogo. Magíster en Políticas Públicas. Investigador del Grupo de Estudios Regionales (GER), Facultad de Ciencias Económicas, Universidad de Antioquia. Correo electrónico: marioalopera19@ hotmail.com - Orcid: 0000-0003-1423-7239 - Google Scholar: https://scholar.google.com/citations?h $\mathrm{l}=\mathrm{es} \&$ user $=\mathrm{T} 6 \mathrm{~K} 2 \mathrm{NcwAAAAJ}$
} 


\title{
Cómo citar este artículo
}

Lopera González, Mario Alfonso. (2020). La formalización minera en el Bajo Caucaantioqueñoatravés delas Áreas de Reserva Especial. Unaaproximaciónal diseño institucional desde el marco de análisis y desarrollo institucional. Estudios Políticos (Universidad de Antioquia), 58. pp. 141-166. DOI: 10.17533/udea.espo.n58a07

\section{A Proposal of Mining Formalization in Antioquia's Bajo Cauca Through the Special Reserve Areas. The Institutional Analysis and Development Framework as an Approach to Institutional Design}

\begin{abstract}
Gold rising prices at the dawn of the 21 st Century have led to a gold rush in Colombia. However, most of the mining in the national territory is made without the minimum legal permits required. Informal gold mining is associated with multiple problems such as resource overuse, environmental externalities, illegal armed groups financing, among others. Thus, public policy as a discipline is called to promote adequate mechanisms for mining formalization. The article depicts an integral

[142] implementation of the Institutional Analysis and Development Framework (IAD) (universal interaction blocks, ADICO syntax, generic rule typology) in analysing, from an institutional design perspective, the context of the Special Reserves Areas in Antioquia's Bajo Cauca. The analysis highlights the utility of the IAD framework for diagnosis and public policy design, and the difficulties that arise from the context and the legal arrangement in trying to use the Special Reserves Areas as an instrument for formalization in the Bajo Cauca.
\end{abstract}

\section{Keywords}

Mining; Mineral Resources Conflicts; Mining Formalization; Institutional Analysis and Development; ADICO Syntax; Colombia. 
La formalización minera en el Bajo Cauca antioqueño a través de las áreas de reserva especial...

\section{Introducción}

La minería carente de los mínimos requisitos legales ha sido una constante en el territorio colombiano. El Censo Minero (Ministerio de Minas y Energía, 2012) señala que $63 \%$ de la minería no se realiza con título, cifra que asciende a $87 \%$ al considerar la minería aurífera. La informalidad en la minería aurífera se asocia con múltiples problemáticas sociales: la generación de externalidades medioambientales, la potencial sobrexplotación del mineral, la evasión de responsabilidades tributarias, entre otras.

Empero, el reto de formalizar la minería artesanal y a pequeña escala (MAPE) aurífera no es solo un desafío regional o nacional, sino que tiene dimensiones globales: países como Ghana, Burundi, República Centro Africana, Zimbabue, Perú y Filipinas son una muestra de aquellos en los que la minería aurífera informal es una problemática apremiante (Grupo de Estudios Regionales, 2018). Los estudios de caso de los países y subregiones son reiterativos en señalar la importancia de políticas públicas que sean sensibles a los contextos locales de los explotadores para lograr el éxito en los procesos de formalización (Hilson et al., 2017; Rochlin, 2018; Spiegel, 2015; Van Bockstael, 2014).

Ante este llamado de atención de la literatura sobre formalización minera, sorprende el hecho de que el marco de análisis y desarrollo institucional (ADI) (Ostrom, 2015), una aproximación especialmente sensible a los contextos específicos de las comunidades, no sea ampliamente utilizado por los investigadores sobre formalización para dar cuenta de su objeto de estudio. Situación que llama aún más la atención al considerar que el ADI se ha mostrado especialmente útil para el estudio de recursos de uso común (RUC) y que, cuando se considera la MAPE aurífera, esta adopta las características de un RUC, tanto por la dificultad en la definición de derechos de propiedad —exclusión- como por la posibilidad, especialmente notable con recursos naturales no renovables, de que haya sobreexplotación —sustracción—; a lo que se añade una posible tragedia: las externalidades medioambientales (Saldarriaga, Villegas y Arango, 2013).

Así las cosas, en el ámbito internacional resalta el trabajo de Natalia Yakovleva y Diego Vásquez (2018), quienes hacen uso del ADI para conceptualizar la estrategia de cooperación entre grandes empresas y MAPE, en tanto alternativa novedosa de gestión de conflictos entre las partes. En el ámbito nacional, el trabajo de Adolfo Eslava (2014) es un referente seminal de 
la implementación del marco ADI en el estudio de la minería aurífera informal; sin embargo, la investigación no se enfocó en los procesos de formalización, sino en las relaciones entre los diversos actores, ya fueran locales, regionales o nacionales, a lo largo de la cadena de valor aurífera.

Estudiosos de las políticas públicas en el contexto local han identificado las potencialidades del marco ADI para diagnosticar múltiples situaciones complejas que se configuran alrededor de la explotación aurífera: apropiación del ethos minero en comunidades no mineras (Silva, 2017), particularidades étnicas de la cadena de comercialización del oro (Valencia, 2014) y garantizar la legalidad en el abastecimiento de oro a los joyeros (Eslava, 2017). Todos estos trabajos tocan de manera tangencial temáticas relacionadas con la formalización de la minería; sin embargo, un análisis concreto de algún mecanismo de formalización, a la luz de las herramientas hermenéuticas, analíticas y conceptuales que el marco ADI brinda, es un pendiente académico que este artículo trata de subsanar a través del análisis de la figura de áreas de reserva especial (ARE) en el Bajo Cauca antioqueño. Además, se hace una aplicación novedosa de la sintaxis atributo, deóntico, objetivo, condiciones, o de lo contrario (sintaxis Adico), respecto a otras implementaciones (Basurto et al., 2010; Siddiki, Weible, Basurto y Calanni, 2011; Watkins y Westphal, 2016), en dos sentidos: primero, incluye en el análisis institucional los grupos armados; segundo, relaciona explícitamente la sintaxis con otros elementos del marco ADI —bloques universales de interacción y la tipología de reglas—.

La investigación se ocupó del Bajo Cauca antioqueño, tanto por su relevancia en la minería en el ámbito nacional como por la altísima informalidad de la subregión. En efecto, entre 2010 y 2017 la subregión dio cuenta, en promedio, de $24 \%$ de la producción aurífera nacional (UPME, s. f.). ${ }^{1}$ Por otro lado, $97 \%$ de las 511 unidades de producción minera (UPM) ${ }^{2}$ censadas entre 2010 y 2014 no contaban con título minero (Consorcio Ambiental Antioqueño, 2010; Proingecon, 2014).

Ahora bien, la selección el mecanismo de ARE responde a la preponderancia que el mecanismo ha ido ganado recientemente. Si bien las ARE existen desde la entrada en vigor del actual código minero, es solo hasta

\footnotetext{
${ }^{1}$ Con un rango que oscila entre $22 \%$ y $30 \%$, y una desviación estándar de 0,02 .

${ }^{2}$ «Hace referencia a un conjunto de labores, con instalaciones, operaciones y/o equipos, que conforman una infraestructura económica y administrativa, dedicada a la explotación de minerales» (Ministerio de Minas y Energía, s. f.).
} 
2013 que se crea una normatividad que especifica el proceso de declaratoria de ARE. En la actualidad, ante la suspensión provisional del Decreto 933 de 2013 (Consejo de Estado, Auto 11001-03-26-000-2014-00156-00 (52506) del 20 de abril de 2016), las ARE son el único mecanismo existente para que los mineros que vienen adelantando sus labores sin contar con título minero puedan hacerse de uno, con excepción de la devolución de áreas (Ley 685 de 2001).

Gráfica 1. Solicitudes de áreas de reserva especial solicitadas en trámite y en vigencia en Antioquia, 2013-2018.

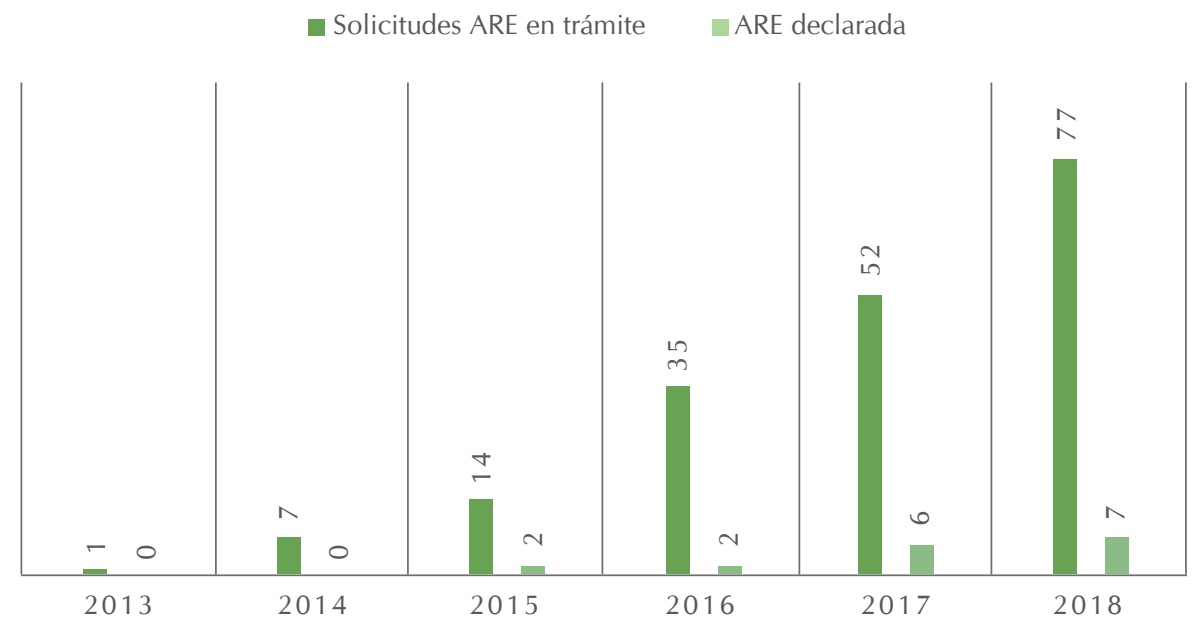

Fuente: elaboración propia a partir de Agencia Nacional de Minería (s. f.).

\section{Marco teórico}

El marco ADI es la propuesta conceptual que Elinor Ostrom ofrece para aproximarse a la estructura de las interacciones humanas y hacer manejable su complejidad inherente. El marco ADI se concibe como un mapa multinivel en el que hay anidamientos conceptuales, cuya utilidad y pertinencia analítica dependen de las preguntas de investigación planteadas.

En el plano más general del marco ADI, se plantea la situación de acción (SA) como la unidad focal de análisis, definiéndola como el espacio social donde los participantes con diversas preferencias interactúan, intercambian bienes y servicios, resuelven problemas, se dominan mutuamente o pelean 
(Ostrom, 2015, pp. 52-53). Tal SA es afectada por tres tipos de variables exógenas: a) las condiciones biofísicas y materiales, que son un conjunto de variables que dan cuenta del entorno y que condicionan los resultados físicamente posibles en una SA dada; b) los atributos de la comunidad, que son los valores de comportamientos aceptados de modo generalizado en la comunidad que se considera; c) las reglas, que son prescripciones sobre lo que se puede, no se puede o se tiene que hacer en un momento dado y que implican vigilancia y sanción en caso de incumplimiento. De la SA emergen resultados evaluables, los cuales retroalimentan las variables exógenas (véase figura 1).

Figura 1. Estructura del marco de análisis y desarrollo institucional, desglose en primer plano de los componentes de la situación de acción y las reglas.

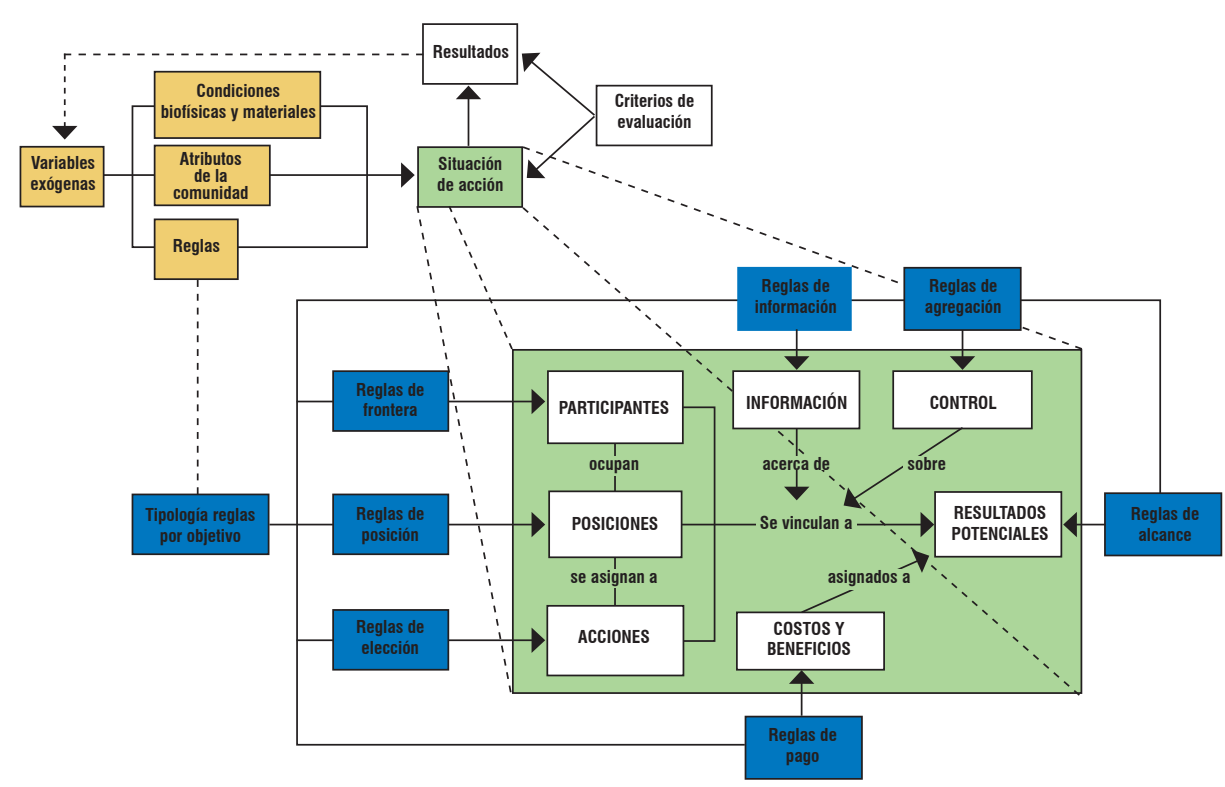

Fuente: elaboración propia a partir de Ostrom (2015).

Al enfocar las SA la propuesta de Ostrom ofrece siete elementos básicos que la configuran y que se relacionan de la siguiente manera: los a) participantes - que pueden ser individuos o cualquiera entre una amplia variedad de entidades organizadas - se asignan a b) posiciones; en estas, los participantes escogen entre c) elecciones, a la luz de la d) información disponible del e) 
La formalización minera en el Bajo Cauca antioqueño a través de las áreas de reserva especial...

control que tienen sobre los vínculos de acción y f) resultados, y de los g) beneficios asignados a acciones y resultados (Ostrom, 2015, p. 256).

Con las tres variables exógenas y los siete elementos que componen una SA quedan enunciados los denominados «bloques de construcción universales de las interacciones humanas» (Ostrom, 2015, p. 43). Sin embargo, aún queda por explorar el pegamento que mantiene unidos estos bloques, aquello que le da consistencia a las interrelaciones. Este es el rol de las instituciones, que son entendidas como las prescripciones que los seres humanos usamos para organizar todas las formas de interacciones repetidas y estructuradas (p. 39).

Los posibles resultados perversos de una SA, o la posibilidad de optimizar resultados, hacen necesario un análisis detallado de las instituciones operantes para poder intervenir o «reparar» tal situación (Ostrom, 2015, p. 255), además de un mecanismo que permita la acumulación de conocimiento. Con estos objetivos en mente se presenta la sintaxis Adico como una herramienta analítica, la cual permite distinguir entre tres tipos de instituciones: estrategias, normas, reglas. La clasificación de la sintaxis consiste en la existencia - $\mathrm{O}$ node cinco elementos: atributo (A), deóntico (D), objetlvo (I), condiciones (C) y o de lo contrario $(\mathrm{O})$. Cuando están presentes los cinco elementos se habla de reglas Adico, cuando solo falte el último elemento se habla de normas ADIC y cuando además falte el deóntico se habla de estrategias AIC.

La sintaxis permite tanto el análisis de «reglas en forma» como de «reglas en uso». Las primeras son fácilmente identificables por su enunciación formal escrita; sin embargo, el grado de influencia en la SA es variable, pues, a priori, no se tiene la certeza de que lo que ordenan estas reglas sean enunciados compartidos y acatados por los participantes. En cambio, las reglas en uso son aquellas que en efecto influyen en el comportamiento de los participantes; empero, dado que estas reglas no necesariamente están escritas, implican mayor grado de dificultad en su sistematización mediante la implementación de la sintaxis (Ostrom, 2015, pp. 236-239).

Además de la sintaxis, se hace una propuesta clasificatoria de reglas que permita identificar cómo los enunciados institucionales analizados afectan la SA que se está estudiando. La tipología de reglas genéricas usa el objetlvo (I) de la sintaxis Adico para identificar el elemento de la SA que se ve afectado directamente por el enunciado institucional; así, a cada parte de la SA corresponde un tipo de regla. 


\section{Marco metodológico}

Se estudia el caso de las ARE en el Bajo Cauca antioqueño usando el marco ADI, con el fin de comprender de manera amplia y estructurada el contexto que condiciona la posibilidad de formalización en la subregión, dado el esquema de anidamiento conceptual que plantea el marco y que permite entrelazar elementos específicos de un nivel analítico con otros de nivel superior. De este modo, la formalización minera, un proceso que desde lo jurídico tiene una apariencia eminentemente técnica, puede ser encuadrado en el contexto histórico, social, territorial y bélico que condiciona su implementación real.

En consecuencia, no se consideraron casos específicos de UPM, sino que se analizó en detalle un mecanismo de formalización —ARE - para evaluar la pertinencia del diseño institucional, dada la configuración institucional existente en la subregión del Bajo Cauca. La información en que se basa el análisis proviene de la triangulación de fuentes.

En la aplicación del análisis institucional hubo dos grandes momentos: en el primero se buscó la reconstrucción de la SA y las variables exógenas de la minería en el Bajo Cauca antiqueño cuando se hace uso del mecanismo de ARE. Para esto se consideraron seis artículos (véase anexo A), ocho entrevistas personales (véase anexo B), cuatro informes, cinco libros, dieciséis noticias, dos tesis de maestría. Esta información fue sistematizada con el software Atlas ti, con una aproximación deductiva que buscaba identificar las categorías analíticas ofrecidas por Ostrom en las diversas fuentes. Si bien la aproximación fue preeminentemente deductiva, la identificación de elementos emergentes de relevancia condujo a complementar la sistematización con categorías de carácter inductivo. Finalmente, se llegó a una sistematización compuesta por seis macrocategorías, que a su vez anidan 39 categorías (véase figura 2).

La preponderancia de los grupos armados organizados al margen de la ley (GAOML) en la configuración institucional de la subregión y la complejidad que implica estudiar la formalización minera en un territorio de soberanía en vilo, instó a una focalización en los GAOML, en aras de mejorar la comprensión de las relaciones de poder históricas en la subregión. Para esto se hizo una revisión parcial de prensa que abarcó un periodo de diez años (2008-2018), en el que se revisaron 62 noticias de diversas fuentes (véase anexo A). Se hizo una sistematización de la información implementando enfoque inductivo de categorización, estableciendo tres categorías independientes y cinco macrocategorías que, a su vez, anidan 44 subcategorías (véase figura 3). 
La formalización minera en el Bajo Cauca antioqueño a través de las áreas de reserva especial...

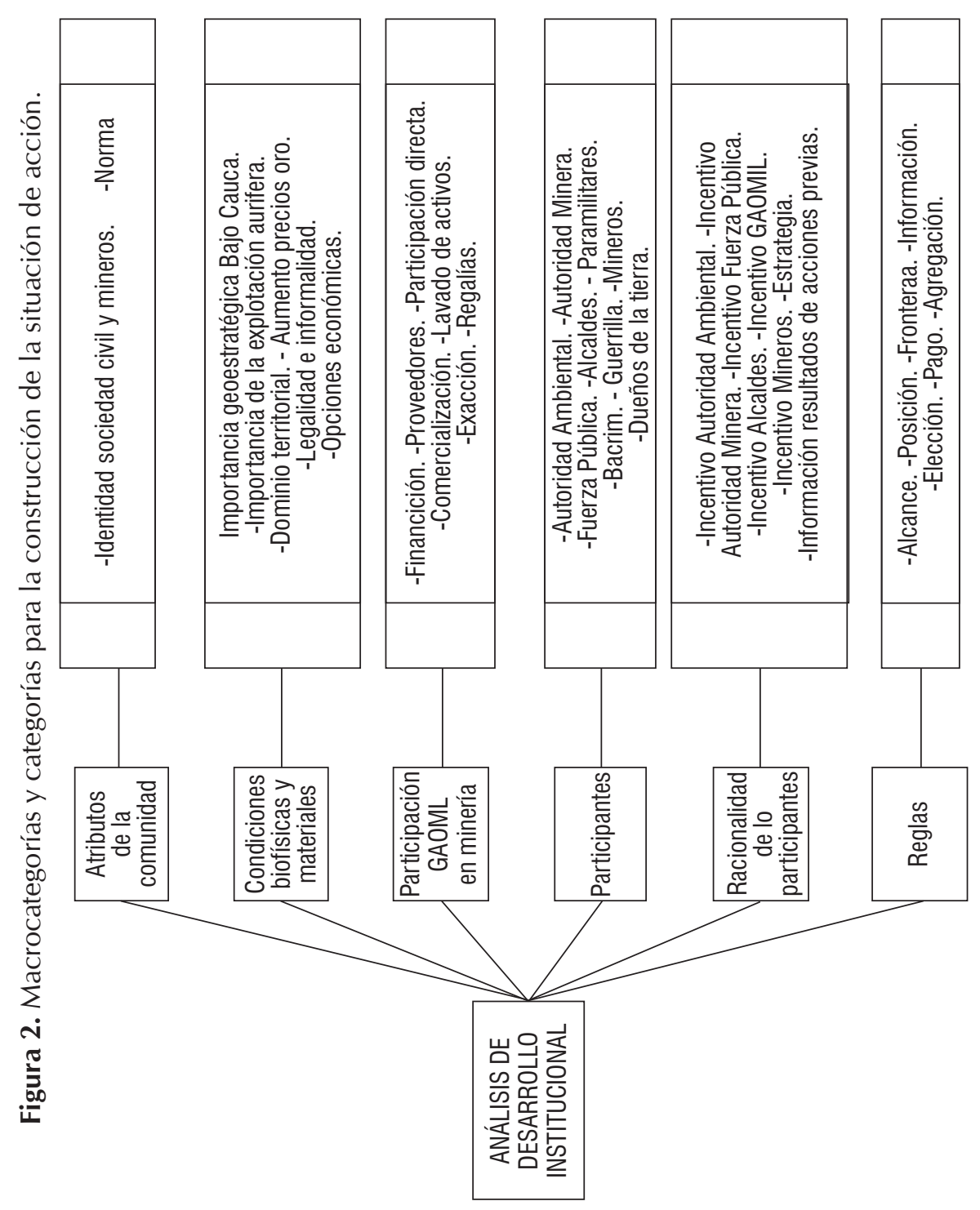

[149]

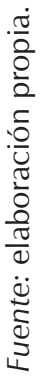


[150]
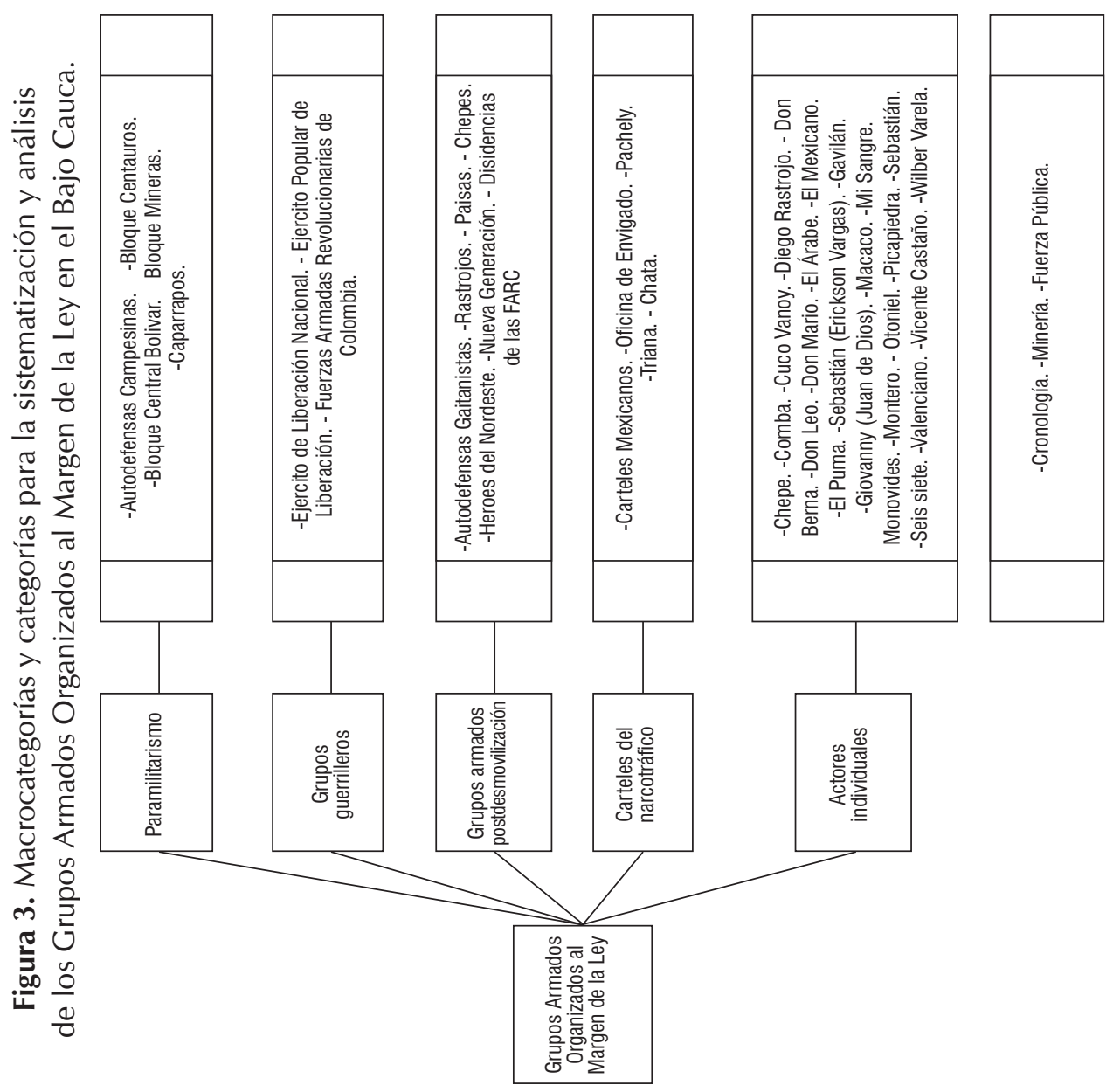

$\frac{0}{0}$
$\frac{0}{0}$
$\frac{0}{2}$
$\frac{0}{U}$
$\frac{\pi}{0}$
$\frac{0}{0}$
$\frac{\pi}{0}$
$\ddot{0}$
$\frac{0}{0}$
$\frac{0}{1}$ 
El segundo momento del análisis institucional fue el de la implementación de la sintaxis Adico y la tipología de reglas generativas. En primer lugar, para dar cuenta de las «reglas en forma», se consideró la legislación minera que atañe a las ARE (véase anexo A); en segundo lugar, para dar cuenta de las «reglas en uso», se consideraron investigaciones atinentes al relacionamiento de los GAOML con la minería en la subregión (véase anexo A). En ambas instancias se construyeron enunciados institucionales y se usó la tipología de reglas genéricas propuesta por Ostrom (2015) para entender el proceso de formalización vía ARE, y así hacerse a una idea sobre su viabilidad en la subregión. Para la implementación de la sintaxis se tomaron elementos de la guía de codificación ofrecida por Ute Brady et al. (2018), pioneros en la implementación de la sintaxis para el análisis de legislaciones (Basurto et al., 2010; Siddiki et al., 2011). También se tomó como referente el trabajo de Cristy Watkins y Lynne Westphal (2016), quienes usan la sintaxis para el análisis de fuentes cualitativas —entrevistas-.

\section{Análisis institucional: variables exógenas}

Este apartado expone las variables exógenas que determinan la SA y reconstruye el escenario de toma de decisión de los mineros informales ante la posibilidad de formalizar su actividad mediante el mecanismo de ARE. EI entrecruzamiento de instituciones formales e informales configura una SA de la que se infieren cuatro resultados potenciales: tres provenientes directamente de la legislación minera y uno de la interacción de los múltiples participantes en el territorio — extracción sin cumplimiento de requisitos legales- (véase figura 4).

\subsection{Condiciones biofísicas y materiales}

El Bajo Cauca antioqueño es una zona de colonización tardía en el territorio nacional. La precariedad de la presencia estatal en sus múltiples manifestaciones — control territorial, infraestructura, prestación de servicios públicos, garantía de derechos - ha tenido como correlato la presencia de GAOML, los cuales se han abrogado y disputado la soberanía en la subregión (García, 1993). Este proceso que se inicia en la década de 1970 por parte de grupos guerrilleros es reconfigurado a finales de 1980 por el paramilitarismo, el cual se consolida y deviene hegemónico hasta su desmovilización en 2006, para ser sucedido por múltiples bandas criminales (Bacrim), grupos guerrilleros y carteles del narcotráfico que continúan disputando y administrando el territorio. 


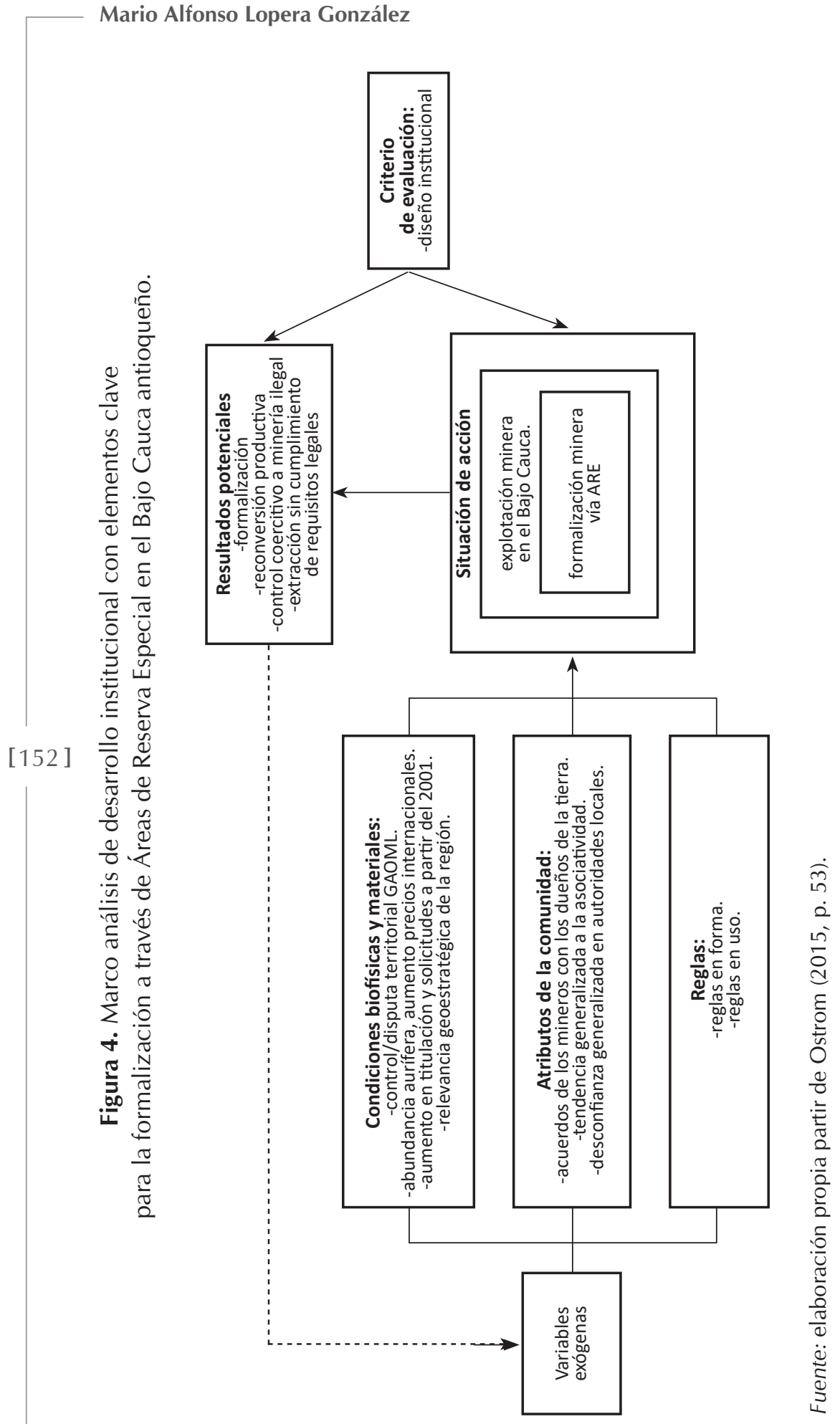


La riqueza aurífera de la región atrajo grandes empresas desde finales del siglo xIx (Poveda, 1981, p. 102); además, con el incremento de los precios del metal al iniciar la década de 1970 se aceleró el parsimonioso proceso de poblamiento territorial, que desde finales de la década de 1930 se venía dando. Asimismo, el repunte de los precios internacionales en la primera década del siglo xxı y su estabilización durante la segunda década explican la preponderancia aurífera de la región, tanto en el plano legal como informal e ilegal (Fundación Ideas para la paz, 2014; Ortiz y Rettberg, 2018).

La entrada en vigor del Código de Minas (Ley 685 de 2001) propició el boom minero nacional. Entre 2002 y 2009 los títulos mineros se incrementaron 274\%, pasando de 2965 a 8126 (Ministerio de Minas y Energía, 2010, p. 69). La subregión no fue ajena a esta fiebre del oro: en 2017 había 166 títulos mineros que abarcaban $23 \%$ del área total de la subregión; de igual modo, a finales de 2017 habían 273 solicitudes de concesión mineras que abarcaban $41 \%$ de la subregión (Gobernación de Antioquia, 2017). Si se tiene en cuenta que los depósitos auríferos no se localizan homogéneamente en el territorio, es de suponer que 36\% del área libre en la subregión no posee la concentración mineral suficiente para hacer atractivo un proyecto minero (comunicación personal, abogado especialista en derecho minero Universidad Externado de Colombia, noviembre 8, 2018), de ahí que la posibilidad de legalizar a los mineros informales vía contrato de concesión resulte difícil.

No solo el oro, sino la localización estratégica de la subregión para los GAOML ha determinado su importancia geopolítica. El Bajo Cauca limita en el Oriente con la serranía de San Lucas y en el Occidente con el Nudo de Paramillo y la serranía de Ayapel. Estos accidentes geográficos aislaban la región haciéndola funcional para la estrategia bélica guerrillera (García, 1993, p. 77). En la década de 1980 el narcotráfico se hizo de grandes extensiones de tierra para el despacho aéreo de cocaína y su procesamiento en laboratorios clandestinos (Uribe, 2001, p. 30); también se introdujo de manera incipiente el cultivo de coca en la subregión. A partir de la década de 1990 se extendió el control sobre toda la cadena de valor de narcóticos por parte de los GAOML, logrando conexiones portuarias con el golfo de Morrosquillo vía la troncal Norte y con el Urabá antioqueño por el departamento del Córdoba.

\subsection{Atributos de la comunidad}

Si bien la historia del Bajo Cauca se remonta al siglo xvı, con la fundación de poblados a causa del ansia española por dar con El Dorado, la importancia 
territorial de Cáceres (1576) decayó. Es en la década de 1930 que surgió el «segundo génesis» del Bajo Cauca (García, 1993, p. 37). Este génesis consistió en un fuerte proceso migratorio de indígenas, negros y campesinos provenientes de zonas como la costa atlántica, Córdoba, Sucre; y la costa pacífica, Chocó y Cauca (García, 2018). Los flujos migratorios se acrecentaron debido al alza aurífera de la década de 1970, atrayendo pobladores con una tradición minera arraigada de las regiones aledañas —Chocó y Córdoba(García, 1993, p. 40).

Al explotar en predios previamente titulados, las relaciones con los dueños de la tierra fueron conflictivas y estos apelaron a las autoridades locales para lograr el desalojo de los mineros o el pago de una renta por explotar el mineral. Los dueños de la tierra hicieron uso de una manifestación local del Estado para imponer de facto un principio que, de jure, estaba prohibido: se impuso la idea de que el predio subía al cielo y bajaba al infierno. El pago de un porcentaje de lo explotado al dueño del predio se normalizó en la región (Giraldo, 2013).

Ahora bien, dada la trashumancia característica de la minería aluvial en la subregión, es posible conjeturar que el tipo de relación internalizada, [154] respecto a los titulares de un terreno, se haya extrapolado a los colonos con predios no titulados cuando el minero cambiaba los sitios de explotación, generándose un sentido de comunidad inusual en un tipo de minería barequeo- que no tiene un particular arraigo con los territorios laborados. En este sentido se interpreta el apoyo en dinero que los mineros aportaron a las grandes movilizaciones regionales de la década de 1980 (García, 1993, p. 116). Por lo demás, la minería no se presentaba como una actividad incompatible con la agricultura. De hecho, lo que primaba en la colonización reciente del Bajo Cauca era un tipo de campesino «todero» que repartía sus actividades de subsistencia entre la agricultura y la minería (Uribe, 2001, p. 15).

De este modo, la minería no resulta ajena al fenómeno clave para la configuración territorial de la subregión: la asociatividad. Sin embargo, no se sugiere la plena confluencia de intereses entre sociedad civil y minería. Es posible identificar conflictos históricos entre mineros mecanizados y pobladores. La aparición de «mineros emergentes» (Giraldo y Muñoz, 2012, p. 76) hizo que no solo la explotación mecanizada fuera preponderante, sino que los conflictos potenciales se acrecentaron. 
El socavamiento de la población civil y la inserción de la región en dinámicas del conflicto armado nacional acentúan problemáticas existentes de poblamiento desorganizado y explotación incontrolada de recurso aurífero (Ministerio del Medio Ambiente, 1995, pp. 11-12), en la medida en que las regulaciones sociales están mediadas por las necesidades económicas y estratégicas de los grupos armados que operan en la zona, los cuales no se corresponden con las necesidades de la población. La cooptación de las agencias estatales por parte de los grupos paramilitares — proceso cuyos jefes denominan para-Estado y que se popularizó con el rótulo de parapolítica (Verdad abierta, 2012, noviembre 1.9) — y su perpetuación a través de las bacrim ha generalizado la desconfianza de la población respecto a las autoridades estatales.

\subsection{Reglas}

En este apartado se hace un acercamiento a los enunciados institucionales que estructuran parcialmente la SA estudiada. Se tiene en cuenta la distinción hecha por Ostrom entre «reglas en forma» y «reglas en uso» (2015, p. 196) (véase tabla 1).

El análisis de reglas realizado mediante la sintaxis Adico y la tipología de reglas permitió detectar varios posibles inconvenientes en la legislación ${ }^{3}$ sobre ARE. Las reglas de frontera, que pueden subdividirse entre las de ingreso aquellas que permiten que un participante ingrese a una posición-y las de salida — aquellas que permiten u obligan a que un participante abandone una posición-, ilustran la dificultad de llegar hasta el final del proceso formalizador. Para los mineros informales se clasificaron siete reglas de ingreso y once reglas de salida. El punto a resaltar es que para llegar a la última fase del proceso, en la cual se firma un contrato de concesión especial y se dispone de la licencia ambiental, se tienen que cumplir con las siete reglas de ingreso, en tanto que basta con que se de una sola de las once reglas de salida para que los mineros tengan que salir del proceso de formalización.

\footnotetext{
${ }^{3}$ El análisis se enfocó primordialmente en la Resolución 546 de 2017; no obstante, el anidamiento legislativo hizo necesario considerar tangencialmente otros cuerpos legislativos (véase anexo A).
} 
Tabla 1. Clasificación de enunciados institucionales.

\begin{tabular}{|c|c|c|c|c|c|c|c|}
\hline & 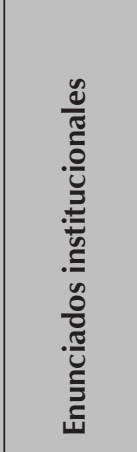 & 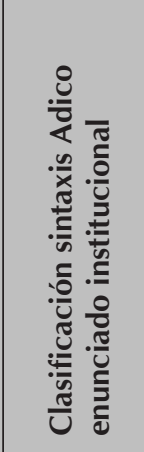 & 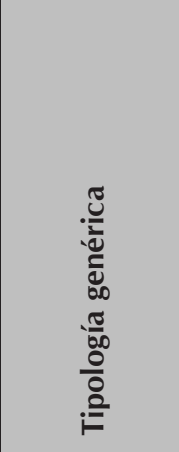 & 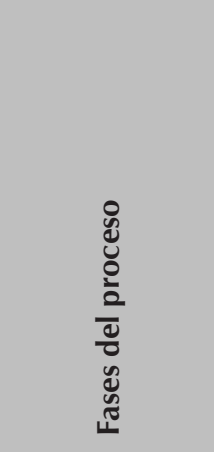 & 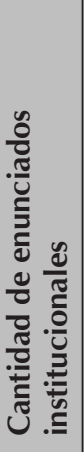 & 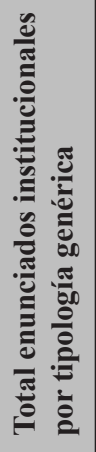 & 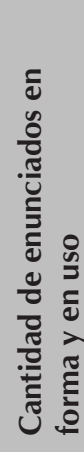 \\
\hline \multirow{21}{*}{$\begin{array}{l}\text { Principales } \\
\text { enunciados } \\
\text { institucionales } \\
\text { que estructuran } \\
\text { la situación de } \\
\text { acción }\end{array}$} & \multirow{18}{*}{ En forma } & \multirow{18}{*}{ Regla } & Alcance & Todas & 1 & 1 & \multirow{18}{*}{88} \\
\hline & & & \multirow{4}{*}{ Posición } & Todas & 2 & \multirow{4}{*}{7} & \\
\hline & & & & Solicitud & 3 & & \\
\hline & & & & ARE declarada & 1 & & \\
\hline & & & & Suscripción & 1 & & \\
\hline & & & \multirow{4}{*}{ Frontera } & Todas & 2 & \multirow{4}{*}{20} & \\
\hline & & & & Solicitud & 8 & & \\
\hline & & & & ARE declarada & 9 & & \\
\hline & & & & Suscripción & 1 & & \\
\hline & & & \multirow{3}{*}{ Información } & Solicitud & 18 & \multirow{3}{*}{33} & \\
\hline & & & & ARE declarada & 11 & & \\
\hline & & & & Suscripción & 4 & & \\
\hline & & & \multirow{3}{*}{ Elección } & Solicitud & 9 & \multirow{3}{*}{21} & \\
\hline & & & & ARE declarada & 9 & & \\
\hline & & & & Suscripción & 3 & & \\
\hline & & & \multirow{2}{*}{ Pago } & ARE declarada & 1 & \multirow{2}{*}{4} & \\
\hline & & & & Suscripción & 3 & & \\
\hline & & & Agregación & ARE declarada & 2 & 2 & \\
\hline & \multirow{3}{*}{ En uso } & \multirow{2}{*}{ Regla } & Frontera & Todas & 3 & 3 & \multirow{3}{*}{11} \\
\hline & & & Pago & Todas & 3 & 3 & \\
\hline & & Estrategia & Elección & Todas & 5 & 5 & \\
\hline
\end{tabular}

Fuente: elaboración propia. 
Respecto a las reglas de información, se encontró el problema de tener que comprobar la tradicionalidad minera ${ }^{4}$ de manera documental, antes de que se haga la visita en campo de verificación de tradicionalidad. La legislación prevé que se presenten al menos dos pruebas documentales de nueve disponibles para dar cuenta de la tradicionalidad minera. Ahora bien, el problema estriba en que dos de estos modos de comprobación son testimonios — sea de vecinos o del alcalde municipal— que dependen precisamente de que estos actores accedan a hacerlo. Además, hay otros cuatro modos — documento de actividad comercial, comprobante de pago de regalías, comprobante de pago de salarios, comprobante de pago de seguridad social - que dan cuenta de un nivel de formalización que no tiene sentido exigir previamente a alguien que intenta ingresar a un proceso de formalización, máxime cuando desde la legislación se reconoce que la minería informal es aquella que no cuenta con ningún tipo de registro contable (Decreto 2191 de 2003). El problema de comprobar la tradicionalidad de manera documental queda ilustrado por el hecho de que este ítem explica 47\% de los 65 rechazos que hubo en Antioquia entre 2016-2018 (Agencia Nacional de Minería, s. f.).

En lo que atañe a las reglas de elección, durante la fase de solicitud, que puede durar hasta ocho meses (véase cuadro 1), se niega la posibilidad de explotar el recurso que brindaría el sustento económico a la comunidad y que representa el sustrato probatorio de la tradicionalidad que evalúa la autoridad minera. ${ }^{5}$ Se genera una situación paradójica en la que el alcalde, que previamente pudo haber certificado la existencia de una comunidad de mineros con explotaciones tradicionales, se ve compelido posteriormente a detener la explotación «ilícita» de dicha comunidad. En la segunda fase está permitida la explotación, pero sin medios mecanizados. En la tercera fase de nuevo se niega la explotación hasta que no se obtenga la licencia ambiental.

\footnotetext{
${ }^{4}$ La Resolución 41107 de 2016 establece sobre las explotaciones tradicionales que es la actividad minera realizada por personas vecinas del lugar que no cuentan con título minero y que por sus características socioeconómicas se constituye en la principal fuente de ingresos de esa comunidad. Las explotaciones mineras deben haber sido ejercidas desde antes de la vigencia de la Ley 685 de 2001, por parte de la comunidad minera solicitante, para lo cual deben acreditar su existencia con un mínimo de dos documentos de cualquier índole que puedan servir de prueba y permitan evidenciar, por parte de la autoridad minera, que son explotaciones tradicionales.

${ }_{5}^{5}$ «Explotaciones Tradicionales: Es la actividad minera realizada por personas vecinas del lugar que no cuentan con título minero y que por sus características socioeconómicas se constituye en la principal fuente de ingresos de esa comunidad [...]» (Resolución 41107).
} 
Cuadro 1. Acciones obligatorias y prohibidas

en el proceso de áreas de reserva especial.

\begin{tabular}{|c|c|c|c|c|c|}
\hline \multicolumn{2}{|c|}{$\begin{array}{c}\text { Fase } 1 \\
\text { Solicitud área de reserva } \\
\text { especial }\end{array}$} & \multicolumn{3}{|c|}{$\begin{array}{c}\text { Fase } 2 \\
\text { Área de reserva especial } \\
\text { declarada }\end{array}$} & $\begin{array}{c}\text { Fase } 3 \\
\text { Contrato especial de } \\
\text { concesión suscrito }\end{array}$ \\
\hline $\begin{array}{c}\text { Envío de } \\
\text { documentación }\end{array}$ & $\begin{array}{l}\text { Visita } \\
\text { técnica de } \\
\text { verificación de } \\
\text { tradicionalidad }\end{array}$ & $\begin{array}{l}\text { Sustracción } \\
\text { de área } \\
\text { de reserva } \\
\text { especial }\end{array}$ & $\begin{array}{l}\text { Estudio } \\
\text { geológico } \\
\text { minero }\end{array}$ & $\begin{array}{l}\text { Plan de } \\
\text { trabajos } \\
\text { y obras }\end{array}$ & $\begin{array}{l}\text { Gestión licencia } \\
\text { ambiental }\end{array}$ \\
\hline \multicolumn{2}{|c|}{$\begin{array}{l}\text { Está prohibido hacer explotación } \\
\text { minera }\end{array}$} & \multicolumn{3}{|c|}{$\begin{array}{c}\text { Está prohibida la implementación } \\
\text { de equipos mecánicos }\end{array}$} & $\begin{array}{l}\text { Está prohibida la } \\
\text { explotación minera }\end{array}$ \\
\hline Hasta 2 meses & Hasta 6 meses & $\begin{array}{l}\text { Hasta } 95 \\
\text { días }\end{array}$ & $\begin{array}{l}\text { Hasta } 2 \\
\text { años }\end{array}$ & $\begin{array}{l}\text { Hasta } 2 \\
\text { años }\end{array}$ & Hasta 9 meses \\
\hline \multicolumn{2}{|c|}{ Hasta 8 meses } & \multicolumn{3}{|c|}{ Hasta 4 años y 95 días } & Hasta 9 meses \\
\hline \multicolumn{6}{|c|}{ Hasta 5 años y 8 meses } \\
\hline
\end{tabular}

Fuente: elaboración propia a partir de la Resolución 546 de 2017.

Finalmente, el análisis permitió reconocer una gran falencia en las reglas

[158] de agregación, pues, dado que se regula una figura colectiva, es necesario explicitar qué pasa con la comunidad minera beneficiaria del ARE cuando uno o varios mineros deciden no hacer lo previsto por la legislación. Esta contempla una serie de situaciones de terminación del ARE. Se afirma, por ejemplo, que la explotación minera se debe llevar a cabo de conformidad con las reglas de seguridad minera y que no hacerlo implica la terminación del ARE; sin embargo, también se establece que el minero que no cumpla será expulsado de la comunidad. Así, si bien todos los miembros de la comunidad minera deben cumplir con la normatividad sobre seguridad minera, el incumplimiento de uno de ellos al respecto no implica la disolución del ARE. Esta claridad, sin embargo, no existe respecto a otras causales de terminación de ARE, como el no pago de regalías, la explotación por fuera del ARE y el incumplimiento de las normas que regulan la comercialización de minerales. Así pues, aún quedan las preguntas: ¿el ARE se termina cuando aquellas obligaciones son incumplidas por un minero, por varios o por todos?

Aparte del análisis de la legislación sobre ARE, también se consideraron las reglas que imponen los GAOML a los mineros de la región — «reglas en 
uso»- - Se encontró que todos los GAOML imponen una regla de frontera consistente en un monto a los mineros por maquinaria ingresada o por el montaje de un nuevo entable que oscila entre 4 y 10 millones de pesos. Además, se identificaron tres reglas de pago: uno mensual de entre $1 \mathrm{y}$ 10 millones de pesos por cada maquinaria que esté operando en la zona; uno de 10\% de lo producido; y uno de 5\% de lo producido por el uso de la tierra para la explotación. Ahora bien, la literatura no esclarece si las dos primeras reglas de pago mencionadas son mutuamente excluyentes o si hay grupos que impongan las dos a los mineros. Respecto a la tercera regla, se identifica que el pago del minero al dueño de la tierra por explotar en sus predios es una práctica específica del Bajo Cauca y que si los GAOML logran adjudicarse la posesión de facto de las tierras este pago correspondería dárselo a ellos; además, dado que la subregión es un territorio disputado, puede darse la situación de que un mismo minero se vea sometido a la exacción de varios GAOML.

\section{Evaluación situación de acción}

Para la viabilidad del diseño institucional de las ARE se considera cada uno de los resultados potenciales previstos por la legislación — formalización, reconversión, control-, además de una posibilidad no incluida en la legislación, pero plausible: la explotación sin contar con los requisitos legales.

El control territorial de GAOML, la poca disponibilidad de áreas libres, sumado a la trashumancia de la minería aluvial son variables exógenas que dificultan el cumplimiento de las reglas de frontera del ARE; además, estar en un proceso de formalización no previene exacciones por parte de los GAOML. De hecho, el pago extorsivo se convierte en la regla más importante que tienen que cumplir los mineros, pues la consecuencia de no pagar es la vida, el desplazamiento o la incautación de la maquinaria.

El trámite administrativo de ARE exige a los mineros el cumplimiento de todas las reglas de frontera de entrada, mientras que basta con que se active una regla de frontera de salida-expulsión para finalizar el proceso; adicionalmente, la presentación de información técnica ajustándose a diversas normatividades en materia ambiental y minera, supone costos en la contratación de profesionales (comunicación personal, investigador grupo IGNEA Universidad Nacional de Colombia, enero 18, 2019; beneficiario ARE en el Bajo Cauca, febrero 1.․, 2019). 
Los beneficios que se desprenden de la figura de ARE son la prerrogativa de explotación, la posibilidad de incluir los estudios geológico-mineros en el Plan de Trabajos y Obras (PTO) y la promesa de capacitación técnica una vez se cuente con el título y la licencia ambiental; la contracara una vez declarada el ARE es la imposibilidad de explotación mientras esta se está delimitando y mientras se tramita la licencia ambiental, lo que se traduce en un costo adicional del proceso: la imposibilidad de generar ganancias. A esto hay que sumar que la prerrogativa de explotación no se extiende a la minería mecanizada, preponderante en el Bajo Cauca. Finalmente, una historia de procesos de formalización fallidos y destrucción de maquinaria ha contradicho las expectativas generadas, reduciendo la confianza hacia el Estado por parte de los mineros (comunicación personal, ingeniero de minas y metalurgia Universidad Nacional de Colombia, enero 16, 2018; investigador grupo IGNEA Universidad Nacional de Colombia, enero 18, 2018).

A partir de lo mencionado, se evidencia que el ARE, además de ser una figura de difícil implementación, no prevé incentivos a los mineros para aplicarla. Más aún, en el Bajo Cauca esta figura implica costos económicos. La posibilidad de buscar una opción económica distinta a la minería depende de las opciones laborales y productivas en la subregión son reducidas (comunicación personal, investigador grupo GEMMA Universidad Nacional de Colombia, agosto 5, 2017). La única alternativa laboral que representa ingresos aproximados a los que procura la minería es el narcotráfico; de hecho, la economía de la región depende de estas dos actividades que llegan a entrelazarse en la comercialización de los minerales. Opciones como la ganadería o el cultivo de caucho no son suficientemente atractivas.

La opción de continuar las operaciones mineras en la ilegalidad tiene varios costos: el pago de vacunas, sobornos a la Policía y la posibilidad latente de que la fuerza pública haga operaciones de destrucción de maquinaria. No obstante, el primero de estos elementos se encuentra presente tanto si hay legalización como si no; por tanto, del pago de extorsiones no depende la decisión de los mineros: esta es una constante. Respecto al pago de sobornos, sí es posible tomarlo como un elemento relevante, de hecho, el único beneficio para iniciar un proceso de formalización es salvarse de la «vacuna verde» (Eslava, 2014, p. 226), faltaría ver en cada caso (UPM) cuánto es el monto y qué tan regular es. Respecto al último elemento, se puede pensar 
que es el disuasivo clave para que el minero no incurra en la extracción ilícita; sin embargo, en la medida en que las autoridades locales encargadas de la detención de la minería sin título se encuentren cooptadas o no cuenten con la capacidad para el cumplimiento de sus funciones, se reduce la efectividad disuasoria.

Se pensaría que una depuración de fuerzas armadas y autoridades administrativas sirve para incrementar la efectividad de este aspecto; sin embargo, esta aproximación de corte coercitivo desconoce la falta de opciones laborales alternativas con ganancias equivalentes, es decir, la demanda laboral en la subregión es inelástica, lo cual es un claro desincentivo a la reconversión económica. A esto se le añade el músculo financiero de los GAOML, dispuestos a financiar la adquisición de equipos (comunicación personal, investigador grupo GEMMA Universidad Nacional de Colombia, agosto 5, 2017). En consecuencia, la apuesta por la coacción, en vez de detener las explotaciones ilícitas, estrecha los vínculos entre mineros y GAOML, y coloca a aquellos en una relación de dependencia respecto a estos.

La situación descrita tiene un efecto social adicional: a la representación negativa que tiene el minero del Estado, por considerarlo destructor de capital y de industria, se añade un elemento legitimador del GAOML (Beltrán, 2015, pp. 16-17), en tanto generador de empleo, al otorgar el crédito para los proyectos mineros (Giraldo y Muñoz, 2012) que las entidades financieras niegan rotundamente bajo el argumento, no infundado, de la alta probabilidad de que el negocio minero esté permeado de criminalidad.

Por otro lado, explotar al margen de la ley tiene incentivos concretos: los mineros no tienen que incurrir en los costos de tiempo y otros recursos para la obtención de permisos legales y los GAOML no interfieren con el proceso de producción (Giraldo y Muñoz, 2012). Desde el punto de vista del minero esto puede facilitar la extracción del mineral en el menor tiempo posible por los medios que él considere pertinentes; no obstante, esta también puede ser la fuente de externalidades negativas para los habitantes que dependen de las fuentes hídricas para su sustento — pesca, agricultura, captación de agua-, provocando tensiones sociales. En consecuencia, es precisamente el resultado potencial que más conflictos genera el que resulta más plausible en la subregión. 


\section{Conclusiones}

La existencia de fallos del mercado justifica el quehacer del Estado mediante políticas públicas que solucionen problemas concretos; no obstante, existen los fallos del Estado y es perfectamente concebible que una intervención estatal que pretendía mejorar una situación la agrave. La falta de información respecto a una situación que se pretende intervenir puede conducir a resultados no deseados. En este sentido, el marco ADI porta una promesa sumamente seductora para los hacedores de políticas públicas y sus analistas: mejorar nuestra capacidad de comprender las instituciones para mejorar su funcionamiento según pasa el tiempo (Ostrom, 2015, p. 71).

El andamiaje analítico y conceptual ofrecido por el marco ADI permitió diseccionar la legislación que regula el mecanismo de ARE y encontrar inconsistencias — que en fases más avanzadas del proceso de formalización se prohíba la explotación que sí se acepta en una fase previa—, vaguedades que en los documentos de «cualquier índole» para probar la tradicionalidad se incluyan registros contables que, por definición, no tienen los mineros informales_, vacíos normativos — falencia en reglas de agregación- y múltiples instancias de veto aplicables por varios participantes —autoridad

[162] minera, autoridad ambiental, alcaldes-, todo lo cual resulta problemático para el mecanismo, en tanto medio hacia la formalización minera.

Al ampliar el análisis a la minería del Bajo Cauca se encontraron múltiples condicionantes: la poca cantidad de áreas libres, la primacía de una minería aluvial mecanizada, acuerdos de arrendamientos con los dueños de la tierra en las que están las minas y la disputa territorial y de rentas por parte de múltiples GAOML. Al cruzar estos elementos con los requerimientos legales para acceder a un ARE se esboza un escenario de altos costos para los que pretenden formalizarse y, por ende, propicio para una minería que explote los recursos sin el cumplimiento de las obligaciones legales.

Las herramientas analíticas del marco ADI —bloques universales de interacción, sintaxis Adico, tipología genérica de reglas- constituyen herramientas poderosas para comprender cómo los diseños institucionales formales pueden funcionar o no en el marco de situaciones de acción gobernadas por condicionantes biofísicos, y de normas y reglas en uso específicas e independientes de las reglas formales. Se considera que la 
promesa del marco ADI se cumple y, en este caso de estudio, sirve para poner en evidencia la dificultad de lograr la formalización de la minería en el Bajo Cauca antioqueño a través de la figura de ARE. Vienen a la mente reminiscencias del campesino kafkiano parado ante la ley, esperando pacientemente a que el guardián lo deje ingresar, pero a diferencia del personaje ficcional los mineros del Bajo Cauca tienen otras opciones a su alrededor, ellos analizan los costos de oportunidad. Lastimosamente, las alternativas no parecen ser las más deseables socialmente, cada instante que el Gobierno demora la elaboración de herramientas efectivas de formalización redunda en una invitación que los guía hacia la ilegalidad.

\section{Referencias bibliográficas}

1. Agencia Nacional de Minería. (s. f.). Áreas de Reserva Especial departamento de Antioquia. Recuperado de https://www.anm.gov.co/sites/default/files/info_areas_ reserva_esp_antio.pdf

2. Basurto, Xavier; Kingsley, Gordon: McQueen, Kelly; Smith, Mshaoni \& Weible, Christopher M. (2010). A Systematic Approach to Institutional Analysis: Applying Crawford and Ostrom's Grammar. Political Research Quarterly, 63 (3), pp. 523-537. https://doi.org/10.1177/1065912909334430

3. Beltrán, Isaac. (2015). Conflicto, construcción de paz y actividad minera en Colombia. Bogotá, D. C.: CREER-IHRB.

4. Brady, Ute et al. (2018). Institutional Analysis of Rules-In-Form Coding Guidelines. CBIE Working Papers. Retrieved from https://complexity.asu.edu/sites/ default/files/papers/cbie_wp_2018-006_0.pdf

5. Colombia. Agencia Nacional de Minería. Resolución 546. (20 de septiembre de 2017). Por la cual se establece el trámite administrativo para la declaración y delimitación de Áreas de Reserva Especial para comunidades mineras. Recuperado de https://www.anm.gov.co/sites/default/files/res_546_20_sep_2017.pdf

6. Colombia. Congreso de la República. Ley 685. (15 de agosto de 2001). Por la cual se expide el Código de Minas y se dictan otras disposiciones. Recuperado de https://www.minambiente.gov.co/images/normativa/leyes/2001/ley_0685_2001.pdf

7. Colombia. Consejo de Estado. Auto 11001-03-26-000-2014-00156-00 (52506). (20 de abril de 2016). Suspensión provisional de efectos Decreto 0933 de 2013. Recuperado de http://www.consejodeestado.gov.co/busquedas/buscadorjurisprudencia

8. Colombia. Ministerio de Minas y Energía. Resolución 41107. (18 de noviembre de 2016). Por la cual se incluyen y modifican algunas definiciones en el Glosario Técnico Minero. Recuperado de https://www.anm.gov.co/sites/default/files/ res_4_1107_18_nov_2016_modifica_glosario_minero.pdf 
9. Colombia. Presidencia de la República. (2003). Decreto 2191. (4 de agosto de 2003). Por el cual se adopta el Glosario Técnico Minero. Recuperado de https://www. funcionpublica.gov.co/eva/gestornormativo/norma.php?i $=9198$

10. Consorcio Ambiental Antioqueño. (2010). Censo Minero de Antioquia. (Informe Final V 1.0).

11. Eslava, Adolfo (ed.). (2014). Oro como fortuna. Instituciones, capital social y gobernanza de la minería aurífera colombiana. Medellín: Colciencias, Universidad Eafit.

12. Eslava, Adolfo. (2017). Análisis institucional de asuntos públicos. El problema de abastecimiento de oro para la joyería en Colombia. Gestión y Política Pública, 26 (1), pp. 253-268.

13. Fundación Ideas para la paz. (2014). Dinámicas del conflicto armado en el Bajo Cauca antioqueño y su impacto humanitario. Recuperado de http://cdn.ideaspaz. org/media/website/document/52efd828c4cbe.pdf

14. García, Clara Inés. (1993). El Bajo Cauca antioqueño. Cómo ver las regiones. Bogotá, D. C.: Cinep.

15. García, Juliana. (2018). Re-configuraciones territoriales emergentes: hacia la consolidación de regiones étnicas estudio de caso Bajo Cauca Antioqueño, Colombia. [Tesis inédita de maestría]. Universidad Nacional de Colombia, Medellín. Recuperado de https://repositorio.unal.edu.co/bitstream/handle/unal/64087/43189188.2018. pdf? sequence $=1$ \&isAllowed $=y$

[164 ] 16.GiraldoRamírez,JorgeyMuñoz,Juan Carlos.(2012). Informalidadeilegalidaden la explotación del oro y la madera en Antioquia. Medellín: Universidad Eafit, Fundación Proantioquia. Recuperado de https://core.ac.uk/download/pdf/47252803.pdf 17. Giraldo Ramírez, Jorge (ed.). (2013). El gobierno del oro en el Bajo Cauca. Una lectura weberiana sobre la explotación. En: Economía criminal y poder político (pp. 33-69). Medellín: Universidad Eafit.

18. Gobernación de Antioquia. (2017). Mapa Minero de Antioquia. Sectorización minera de los municipios 2017. Recuperado de https://issuu.com/gobantioquia/docs/ mapa_minero_de_antioquia

19. Grupo de Estudios Regionales. (2018). Valoración de la Actividad Sectorial de la Minería Artesanal y en Pequeña Escala MAPE Para su Abordaje en el Informe EITI. Medellín. Manuscrito inédito.

20. Hilson, Gavin \& Maconachie, Roy. (2017). Formalising Artisanal and SmallScale Mining: Insights, Contestations and Clarifications. Area, 49 (4), pp. 443-451. https://doi.org/10.1111/area.12328

21. Hilson, Gavin; Hilson, Abigail; Maconachie, Roy; McQuilken, James \& Goumandakoye, Halima. (2017). Artisanal and Small-Scale Mining (ASM) in SubSaharan Africa: Re-Conceptualizing Formalization and «illegal» Activity. Geoforum, 83, pp. 80-90. https://doi.org/10.1016/j.geoforum.2017.05.004 
La formalización minera en el Bajo Cauca antioqueño a través de las áreas de reserva especial...

22. Ministerio de Minas y Energía. (2010). Sector Minas. En: Memorias al Congreso de la República 2009-2010 (pp. 63-98). Recuperado de https://www.minenergia. gov.co/documents/10180/614096/01-intoduccion.pdf/3fa24d6e-8439-4744-8f83ae43ac1585ba

23. Ministerio de Minas y Energía. (2012). Censo Minero Departamental 2010-2011. Bogotá, D.C.: Colombia. Recuperado de https://www.minenergia. gov.co/documents/10180/698204/CensoMinero.pdf/093cec57-05e8-416b-8e0c$5 \mathrm{e} 4 \mathrm{f} 7 \mathrm{c} 1 \mathrm{~d} 6820$

24. Ministerio de Minas y Energía. (s. f.). Plan de Acción de Formalización. Recuperado de https://asogravas.org/wp-content/uploads/2017/11/Plan-deAcci $\%$ C3\%B3n-Formalizaci\%C3\%B3n-.pdf

25. Ministerio del Medio Ambiente. (1995). Visita de seguimiento zona minera Bajo Cauca. (Informe técnico).

26. Ortiz-Riomalo, Juan Felipe y Rettberg, Angelika. (2018). Minería de oro, conflicto y criminalidad en los albores del siglo xxı en Colombia: perspectivas para el posconflicto colombiano. Colombia Internacional, 93, pp. 17-63. https://doi. org/10.7440/colombiaint93.2018.02

27. Ostrom, Elinor. (2015). Comprender la diversidad institucional. México, D.F.: FCE.

28. Poveda, Gabriel. (1981). Minas y mineros de Antioquia. Medellín: Banco de la República.

29. Proingecon LTDA. (2014). Identificación y caracterización de la actividad minera en municipios priorizados que no se incluyeron en el censo 2010-2011. (Informe técnico).

30. Rochlin, James. (2018). Informal Gold Miners, Security and Development in Colombia: Charting the Way Forward. Extractive Industries and Society, 5 (3), pp. 330-339. https://doi.org/10.1016/j.exis.2018.03.008

31. Saldarriaga-Isaza, Adrián; Villegas-Palacio, Clara \& Arango, Santiago. (2013). The Public Good Dilemma of a Non-Renewable Common Resource: A Look at the Facts of Artisanal Gold Mining. Resources Policy, 38 (2), pp. 224-232. https://doi. org/10.1016/j.resourpol.2013.02.001

32. Siddiki, Saba; Weible, Christopher; Basurto, Xavier \& Calanni, John. (2011). Dissecting Policy Designs: An Application of the Institutional Grammar Tool. Policy Studies Journal, 39, pp. 79-103. https://doi.org/10.1111/j.1541-0072.2010.00397.x

33. Silva, Santiago. (2017). Minería aurífera informal e imposición de arreglos institucionales incumplidores: análisis institucional del caso de Buriticá, Antioquia, en 2009-2014. Opera, 20, pp. 157-178. https://doi.org/10.18601/16578651.n20.08

34. Spiegel, Samuel J. (2015). Shifting Formalization Policies and Recentralizing Power: The Case of Zimbabwe's Artisanal Gold Mining Sector. Society and Natural Resources, 28 (5), pp. 543-558. https://doi.org/10.1080/08941920.2015.1014606 
35. Unidad de Planeación Minero Energética (UPME). (s. f.). Oro. Producción. Tableau Public. Recuperado de https://public.tableau.com/profile/upme\#!/vizhome/ Oro_1/Historia1

36. Uribe de Hincapié, María Teresa. (2001). Desplazamiento forzado en Antioquia 1985-1998. Vol. 1. Bogotá, D. C.: Conferencia Episcopal Colombiana.

37. Valencia, Ingrid. (2014). Minería informal y capital social. Criterios orientadores para la formalización de la Pequeña Minería en el Departamento del Chocó. [Tesis inédita de maestría]. Universidad Eafit, Medellín. Recuperado de https://repository.eafit.edu.co/bitstream/handle/10784/5442/IngridValencia_2014. pdf? sequence $=2 \&$ isAllowed $=y$

38. Van Bockstael, Steven. (2014). The Persistence of Informality: Perspectives on the Future of Artisanal Mining in Liberia. Futures, 62, pp. 10-20. https://doi. org/10.1016/j.futures.2014.02.004

39. Veiga, Marcello M. \& Marshall, Bruce G. (2018). The Colombian Artisanal Mining Sector: Formalization is a Heavy Burden. The Extractive Industries and Society, 6 (1), pp. 223-228. https://doi.org/10.1016/j.exis.2018.11.001

40. Verdad Abierta. (2012, noviembre 1.). El libreto de la parapolítica según Mancuso, «El Alemán» y «Jorge 40». Recuperado de https://verdadabierta.com/laparapolitica-segun-mancuso-el-aleman-y-jorge-40/

41. Watkins, Cristy \& Westphal, Lynne M. (2016). People don't Talk in Institutional Statements: A Methodological Case Study of the Institutional Analysis

[166] and Development Framework. Policy Studies Journal, 44, pp. 98-122. https://doi. org/10.1111/psj.12139

42. Yakovleva, Natalia \& Vazquez-Brust, Diego Alfonso. (2018). Multinational Mining Enterprises and Artisanal Small-Scale Miners: From Confrontation to Cooperation. Journal of World Business, 53, pp. 52-62. https://doi.org/10.1016/j. jwb.2017.08.004 